\title{
Das Betrachten des Übersetzungsprozesses: primäre Ergebnisse einer Fallstudie in Leipzig
}

\author{
[Observation of the translation process: initial results of a case study \\ carried out in Leipzig] \\ http://dx.doi.org/10.11606/1982-88372340140
}

\author{
Luciane Leipnitz $^{1}$ \\ Minka Beate Pickbrenner ${ }^{2}$
}

\begin{abstract}
A case study was carried out in 2018 in Leipzig, resulting in a research on the translation competence of undergraduate students that followed the PACTE Group's methodology (2003, 2008, 2011B, among others). Data were collected for comparison with some studies developed in Brazil (LIPARINI CAMPOS \& LEIPNITZ, 2017; LIPARINI CAMPOS, LEIPNITZ \& BRAGA, 2017; LIPARINI CAMPOS, BRAGA \& LEIPNITZ, 2015). The study comprised the translation of a specialized text (around 200 words) into the students' mother language. Online texts of medical journals in three languages (German, English and Portuguese) were selected. Twenty-three study participants translated the source texts into their respective mother languages (German, English, Spanish or Portuguese) and answered three questionnaires (about their translator's profile, conceptions on translation, and translation problems). These different methodologies of analysis were evaluated with triangulation, thus investigating the translation process of students in training. Data from this case study conducted at the Institut für Angewandte Linguistik und Translatologie (IALT - Institute of Applied Linguistics and Tradutology), in Leipzig, were collated with those studies accomplished in Brazil, with the aim of qualifying translation didactics in undergraduate courses. The initial results of this case study are shown and discussed here
\end{abstract}

Keywords: translation process; translation competence; didactics of translation.

Resumo: Durante um período de estudos em Leipzig em 2018 foi desenvolvida uma pesquisa sobre a competência tradutória de estudantes. A pesquisa foi conduzida de acordo com as metodologias do Grupo PACTE (2003, 2008, 2011b, dentre outros) e foram levantados dados para uma análise comparativa com pesquisa desenvolvida no Brasil (LIPARINI CAMPOS, LEIPNITZ 2017; LIPARINI CAMPOS, LEIPNITZ, BRAGA 2017; LIPARINI CAMPOS, BRAGA, LEIPNITZ 2015). O estudo compreendeu a tradução de um texto especializado curto (cerca de 200 palavras) para a língua materna. Foram escolhidos textos de revistas médicas disponíveis online em três línguas de partida (alemão, inglês e português). Os 23 participantes do estudo traduziram o texto de

\footnotetext{
${ }^{1}$ Universidade Federal da Paraíba, Departamento de Mediações Interculturais, Cidade Universitária, Campus I, Conjunto Humanístico, Bloco A, João Pessoa, PB, 58051-900, Brasil. E-mail: luciane.leipnitz@gmail.com. ORCID: 0000-0002-7425-2089

${ }^{2}$ Secretaria de Desenvolvimento Econômico e Turismo, Departamento de Promoção Comercial e Assuntos Internacionais, Av. Borges de Medeiros, 1.501, Centro Histórico, Porto Alegre, RS, 90119-900, Brasil. Email: minkapick@gmail.com. ORCID: 0000-0002-3757-1312
}

\section{(cc) BY-NC}

Pandaemonium, São Paulo, v. 23, n. 40, mai.-ago. 2020, p. 140-165 
Leipnitz, L.; Pickbrenner, M.B. - Das Betrachten des Übersetzungsprozesses

partida para suas línguas maternas (alemão, inglês, espanhol e português) e responderam a três questionários (sobre o perfil do tradutor, sobre a sua concepção sobre tradução e sobre os problemas tradutórios). As diferentes metodologias de análise serão avaliadas em triangulação, visando investigar o processo tradutório de estudantes em formação. Os dados desse estudo de caso no Instituto de Linguística Aplicada e Tradutologia (IALT) em Leipzig serão contrastados com o estudo realizado no Brasil com o objetivo de qualificar a didática da tradução nos cursos de graduação. Serão apresentados e discutidos aqui os resultados iniciais desse estudo de caso.

Palavras-chave: processo tradutório; competência tradutória; didática da tradução.

Zusammenfassung: Während eines Forschungsaufenthalts in Leipzig 2018 wurde eine Studie über die Übersetzungskompetenz von StudentInnen entwickelt. Die Forschung wurde nach den Methoden der PACTE-Gruppe (2003, 2008, 2011B, u.a.) durchgeführt, indem Daten für eine komparative Analyse mit Ergebnissen einer Studie, die in Brasilien entwickelt wurde (LIPARINI CAMPOS, LEIPNITZ 2017; LIPARINI CAMPOS, LEIPNITZ, BRAGA 2017; LIPARINI CAMPOS, BRAGA, LEIPNITZ 2015), erhoben wurden. Die Studie bestand aus der Übersetzung eines kurzen Fachtextes (mit ca. 200 Wörtern) in die Muttersprache. Es wurden Texte aus medizinischen Online-Fachzeitschriften in drei verschiedenen Ausgangssprachen (Deutsch, Englisch und Portugiesisch) ausgewählt. Die 23 TeilnehmerInnen des Experimentes haben den Ausgangstext in ihre Muttersprache (Deutsch, Englisch, Portugiesisch oder Spanisch) übersetzt. Außerdem wurden drei Fragebögen über das Profil der ÜbersetzerInnen, über die Übersetzungskonzeption der ÜbersetzerInnen und über die Übersetzungsprobleme beantwortet. Die verschiedenen Analysemethoden sollen durch Triangulation bewertet werden, um den Übersetzungsprozess der StudentInnen in der Ausbildung zu untersuchen. Die Daten dieser Fallstudie am Institut für Angewandte Linguistik und Translatologie (IALT) in Leipzig sollen mit den Ergebnissen der Studie in Brasilien kontrastiert werden, um zur Verbesserung der Übersetzungsdidaktik in Übersetzungsstudiengängen beizutragen. In diesem Artikel werden zunächst primäre Ergebnisse der Fallstudie präsentiert und diskutiert.

Stichwörter: Übersetzungsprozess; Übersetzungskompetenz; Übersetzungsdidaktik.

\section{Einleitung}

Während eines Forschungsaufenthalts am Institut für Angewandte Linguistik und Translatologie (IALT) in Leipzig 2018 wurde eine Studie zur die Übersetzungskompetenz von StudentInnen, künftigen ÜbersetzerInnen, entwickelt. Mit dieser Forschung, die nach den Methoden der Studien der PACTE ${ }^{3}$-Gruppe (2003, 2008, 2011b, u.a.) durchgeführt wurde, wurden neue Daten erhoben, um eine komparative Analyse mit einer Studie in Brasilien (LIPARINI CAMPOS, LEIPNITZ 2017; LIPARINI Campos, Leipnitz, Braga 2017; Liparini Campos, Braga, LeIPNitz 2015) zu realisieren.

\footnotetext{
3 PACTE (Procés d'Adquisició de la Competència Traductora i Avaluació) ist der Name einer Foschungsgruppe der Autonomen Universität Barcelona, die seit 1997 empirische und experimentelle Studien über die Aneignung von Übersetzungskompetenz entwickelt. Für weitere Informationen siehe $<$ http://grupsderecerca.uab.cat/pacte/en> (17/09/2019).
}

Pandaemonium, São Paulo, v. 23, n. 40, mai.-ago. 2020, p. 140-165 
Leipnitz, L.; Pickbrenner, M.B. - Das Betrachten des Übersetzungsprozesses

Um den Übersetzungsprozess der StudentInnen in der Ausbildung zu untersuchen, wurden die Daten beider Studien - in Brasilien 2014-2016 und in Deutschland 2018 anhand der Verwendung von unterschiedlichen Methoden erhoben. Die Ergebnisse der komparativen Analyse dieser Studien sollen den ForscherInnen (DozentInnen und ProfessorInnen an den Universitäten) neue Arbeitsperspektiven in Richtung auf eine qualifiziertere Ausbildung der ÜbersetzerInnen bieten.

Schon seit mehr als 20 Jahren werden Studien über den Übersetzungsprozess an unterschiedlichen Forschungszentren (vgl. u. a. PACTE 2003, 2008, 2011b; GoNÇALVES 2003, 2005, 2015; GÖPFERICH 2008, 2009) entwickelt. Aus einer neuen Perspektive in Translation Studies betrachten die ForscherInnen nicht nur das Übersetzungsprodukt, sondern die Übersetzung als einen Prozess, der durch die Benutzung von unterschiedlichen Forschungsmethoden und modernen Softwares untersucht werden soll. Anhand der Datentriangulation bieten neue Technologien (z.B. Schreib-LoggingSoftware, Screen-Recording-Software, Eye-Trackers, u.a.) effizientere Analysen und eine solidere Basis für Diskussionen über Forschungsergebnisse. Mithilfe von modernen Studien über die Entwicklung der Übersetzungskompetenz versuchen ForscherInnen, neue und effizientere Methoden für die Ausbildung als ÜbersetzerInnen in verschiedenen Universitäten zu entwickeln. Die Ergebnisse solcher Forschungen sollen die Didaktik in den Übersetzungsstudiengängen verbessern, um kompetentere ÜbersetzerInnen auszubilden.

Mit diesem Ziel haben ForscherInnen an der Universität in João Pessoa (Brasilien) eine longitudinale Studie (2014-2016) entwickelt. In drei verschiedenen Semestern wurde der Übersetzungsprozess der StudentInnen im Studiengang „Bacharelado em Tradução“ anhand drei verschiedener Übersetzungsaufgaben und unter unterschiedlichen Betrachtungsmitteln untersucht. Die ForscherInnen, DozentInnen im erwähnten Studiengang, bezweckten damit, die Inhalte im Studienplan effizienter zu gestalten, die Autonomie der StudentInnen zu entwickeln und qualifiziertere ÜbersetzerInnen auf den Arbeitsmarkt vorzubereiten.

Die Fallstudie in Leipzig, deren Daten mit den Ergebnissen der Studie in Brasilien kontrastiert werden sollen, wurde 2018 am Institut für Angewandte Linguistik und Translatologie (IALT) durchgeführt. Im Folgenden werden die primären Ergebnisse der Fallstudie in Leipzig präsentiert und diskutiert. 


\section{Das Forschungsprojekt in Brasilien}

Übersetzung ist laut der PACTE-Gruppe (2003) ein kommunikativer Vorgang und Übersetzungskompetenz ein Erkenntnissystem der zugrundeliegenden und nötigen Fähigkeiten, um Übersetzungsaufgaben auszuführen. Um Forschungen über den Erwerb von Übersetzungskompetenz durchzuführen, hat die Forschungsgruppe das Übersetzungskompetenz-Modell (ÜK-Modell) (PACTE 2003) entwickelt.

Das ÜK-Modell (PACTE 2003) besteht aus psychophysiologischen Komponenten und fünf Subkompetenzen: (1) der zweisprachigen Subkompetenz, (2) der extralinguistischen Subkompetenz, (3) der Übersetzungskonzeption, (4) der instrumentellen Subkompetenz und (5) der strategischen Subkompetenz.

Abb. 1: Holistisches Übersetzungskompetenz-Modell (vgl. PACTE 2003)

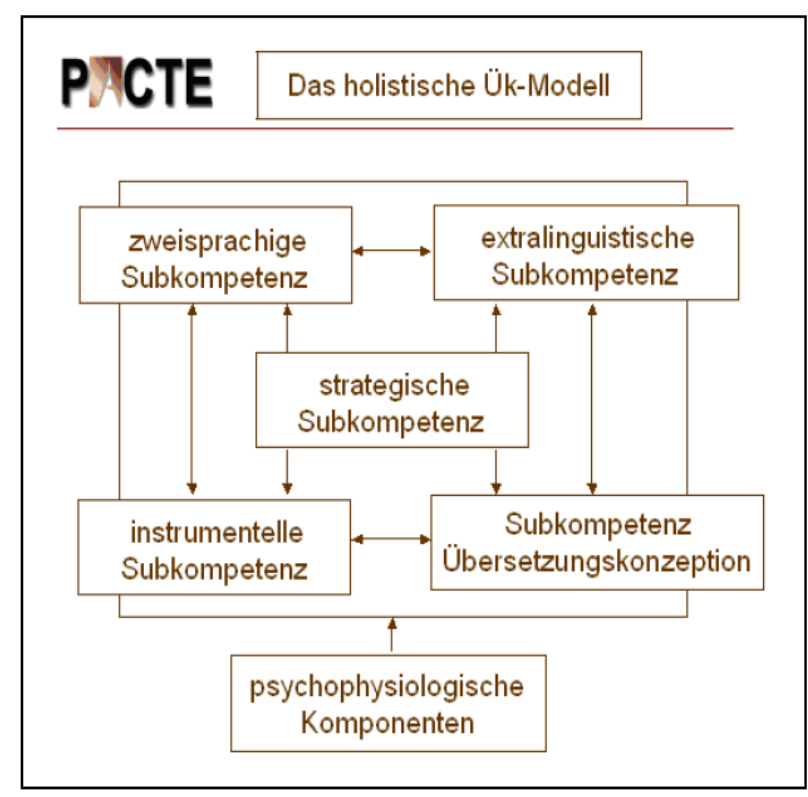

Nach den Studien der PACTE-Gruppe sind die zweisprachigen und extralinguistischen Subkompetenzen keine spezifischen Kompetenzen der ÜbersetzerInnen, sondern allgemeine Kompetenzen mehrsprachiger/zweisprachiger Menschen (HuRTAdo ALBIR 2005). Die psychophysiologischen Komponenten gehören zu den Expert-Kenntnissen beim Übersetzen (HuRTADO AlBIR 2005). Die instrumentellen und strategischen Subkompetenzen und die Übersetzungskonzeption sind 
Leipnitz, L.; Pickbrenner, M.B. - Das Betrachten des Übersetzungsprozesses

andererseits spezifische Subkompetenzen der ÜbersetzerInnen, die nur im Übersetzungsprozess identifiziert werden können.

Um die Übersetzungsdidaktik im Studiengang „Bacharelado em Tradução“4 an der Bundesuniversität in João Pessoa $\left(\mathrm{UFPB}^{5}\right)$ qualitativ zu verbessern, wurde eine longitudinale Studie über die Übersetzungskompetenz der StudentInnen im Laufe des Studiengangs durchgeführt. Das Forschungsprojekt „Die Übersetzungskompetenz und Ausbildung der ÜbersetzerInnen: die Entwicklung der spezifischen Subkompetenzen der ÜbersetzerInnen“ (Prof. Dra. Tania Liparini Campos mit Unterstützung des CNPq 485158/203-2) wurde nach den Forschungsergebnissen der PACTE-Gruppe im Studiengang (2014-2016) in verschiedenen Semestern durchgeführt: am Anfang, in der Mitte und am Ende der Ausbildung. Nach der Datenerhebung haben die ForscherInnen anhand der Ergebnisse die Übersetzungskonzeption dieser StudentInnen untersucht (LiPARINI CAMPOS, LEIPNITZ 2017; LIPARINI CAMPOS, LEIPNITZ, BRAGA 2017; LIPARINI CAMPOS, BRAGA, LEIPNITZ 2015).

Für die Datenerhebung wurden von den StudentInnen drei verschiedene kurze Texte (ca. 200 Wörter) übersetzt. Die Übersetzungsaufgaben wurden im Computerraum unter Benutzung der Softwares Translog ${ }^{6}$ und Camtasia Studio ${ }^{7}$ durchgeführt. Mit den Softwares wurde der Übersetzungsprozess während der Lösung einer Übersetzungsaufgabe untersucht. Die ForschungsteilnehmerInnen haben zusätzlich drei verschiedene Fragebögen (über das Profil der ÜbersetzerIn, über die Übersetzungskonzeption und über die Übersetzungsprobleme/-schwierigkeiten beim Übersetzen) beantwortet.

Der Fragebogen „Profil der ÜbersetzerInnen“ enthielt persönliche Fragen, wie z.B. Alter der StudentInnen, Semester im Studiengang, Sprachkenntnisniveau, Auslandsaufenthalt, berufliche Erfahrung, usw. Laut der Antworten waren die TeilnehmerInnen der Studie in João Pessoa zwischen 19 und 31 Jahre alt und nur ein(e) StudentIn hatte bereits einen anderen Studiengang (Jura) abgeschlossen. Die TeilnehmerInnen nannten sich „kompetent“ und „,wenig kompetent“ in Englisch, ihrer ersten Fremdsprache im Studiengang, und nur eine StudentIn informierte, sie hätte zwei

\footnotetext{
${ }^{4}$ Curso de Bacharelado em Tradução (UFPB) <http://www.cchla.ufpb.br/ctrad/> (22/11/2019).

${ }^{5}$ Universidade Federal da Paraíba (UFPB) <https://ufpb.br/> (17/09/2019).

${ }^{6}<$ https://sites.google.com/site/centretranslationinnovation/translog-ii $>(17 / 09 / 2019)$.

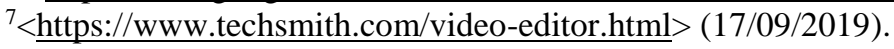


Leipnitz, L.; Pickbrenner, M.B. - Das Betrachten des Übersetzungsprozesses

Monate in Kanada gelebt. Alle TeilnehmerInnen berichteten, sie hätten noch keine andere berufliche Erfahrung.

Die Methoden der PACTE-Gruppe (2005a, 2005b, 2008) gehen davon aus, dass die Entwicklung jeder Übersetzungssubkompetenz durch spezifische Variablen (u.a. den Entscheidungsfindungsprozess und die Identifizierung der Übersetzungsprobleme) und durch unterschiedliche Mittel (Fragebögen, Softwares, Lautprotokolle, usw.) untersucht werden muss. Alle Variablen sollen mit der Bewertung der Textproduktionen beim Übersetzen (Datentriangulation) verglichen werden.

Mithilfe eines Fragebogens über die Übersetzungskenntnisse können die ForscherInnen das Übersetzungskonzept der ÜbersetzerInnen als dynamisch oder statisch auswerten. Laut der Ergebnisse der Studie von PACTE (2011b) zeigen die ÜbersetzerInnen ein statisches Übersetzungskonzept, wenn sie die Übersetzungsaufgabe als einen sprachlichen Prozess wahrnehmen. Am wichtigsten in der Zieltextproduktion dieser ÜbersetzerInnen sind die strukturellen Aspekte des Textes. Wenn die ÜbersetzerInnen die Übersetzungsaufgabe als eine textuelle, kommunikative und funktionelle Aktivität verstehen, zeigen sie ein dynamisches Übersetzungskonzept.

Die Studien von PACTE (2008) zeigten, dass sich die Äußerungen der ÜbersetzerInnen durch dynamische Konzepte und die der SprachlehrerInnen durch statische Konzepte bezeichnen lassen können. Laut den PACTE-ForscherInnen standen diese Ergebnisse im Einklang mit den unterschiedlichen Ausbildungen der TeilnehmerInnen.

Die Entwicklung der Übersetzungskompetenz ist ein nicht linearer und dynamischer Prozess (PACTE 2000), der sich von der Vorkompetenz zur Übersetzungskompetenz entwickelt und durch die Variablen Dynamischer Index und Kohärenz-Koeffizient gemessen werden kann. Der Dynamische Index pendelt zwischen -1 und +1 . Je näher der Index bei -1 liegt, desto statischer ist das Übersetzungskonzept, je näher er bei +1 liegt, desto dynamischer ist das Übersetzungskonzept. Ein Index gegen Null bedeutet Ambivalenz im Übersetzungskonzept. Für den Kohärenz-Koeffizienten gelten die Werte 0, 0,5 und 1,0. Der 1,0 Koeffizient bedeutet ein konsistenteres Übersetzungskonzept, der Null-Koeffizient bedeutet ein inkonsistentes Übersetzungskonzept. 
Leipnitz, L.; Pickbrenner, M.B. - Das Betrachten des Übersetzungsprozesses

Den Daten der Forschung in João Pessoa gemäß zeigten die StudentInnen am Anfang des Studiengangs, der ersten Phase der Forschung (2014), eine gering entwickelte Übersetzungskompetenz und ein pendelndes Übersetzungskonzept (zwischen statisch und dynamisch).

In der zweiten Phase der Forschung (2015) zeigten sie ein dynamischeres Übersetzungskonzept, d.h., dass durch den Besuch von Lehrveranstaltungen in einem Zeitraum von 20 Monaten eine Entwicklung der Übersetzungskonzeption der StudentInnen beobachtet wurde (LIPARINI CAMPOS, LEIPNITZ 2017).

Tab.1: Dynamischer Index und Kohärenz-Koeffizient nach der Übersetzungskonzeption der StudentInnen im Vergleich in den beiden Phasen der Studie in Brasilien (LIPARINI CAMPOS, LEIPNITZ 2017) ${ }^{8}$

\begin{tabular}{|l|l|l|}
\hline & $\begin{array}{l}\text { Erste Phase der } \\
\text { Studie (2014) }\end{array}$ & $\begin{array}{l}\text { Zweite Phase der } \\
\text { Studie (2015) }\end{array}$ \\
\hline $\begin{array}{l}\text { Durchschnittlicher Dynamischer } \\
\text { Index }\end{array}$ & 0,05 & 0,4 \\
\hline $\begin{array}{l}\text { Durchschnittlicher Kohärenz- } \\
\text { Koeffizient }\end{array}$ & 0,25 & 0,38 \\
\hline
\end{tabular}

Die Ergebnisse der Forschung in Brasilien werden noch mit weiteren Experimenten und TeilnehmerInnen konfrontiert. In dem Zeitraum von 2014-2015 zeigten aber die schon systematisierten Ergebnisse einen sichtbaren Fortschritt der Übersetzungskonzeption der StudentInnen im Studiengang in João Pessoa.

\section{Die Studie in Leipzig}

Im Sommersemester 2018 wurde am Institut für Angewandte Linguistik und Translatologie an der Universität in Leipzig eine neue Fallstudie zur Übersetzungskompetenz der StudentInnen durchgeführt. Mit dieser neuen Forschung, die nach der Methode der Studie in Brasilien strukturiert wurde, wird beabsichtigt, eine kontrastive Analyse der Daten von beiden Studien - in Brasilien und in Deutschland durchzuführen. Durch die Erhebung der Daten zu den Ähnlichkeiten und Unterschieden

\footnotetext{
${ }^{8}$ Diese und alle weiteren Tabellen wurden von den Autorinnen selbst erstellt.
} 
Leipnitz, L.; Pickbrenner, M.B. - Das Betrachten des Übersetzungsprozesses

bezüglich der Profile und Übersetzungskenntnisse der StudentenInnen in unterschiedlichen Übersetzungsstudiengängen hat diese kontrastive Studie als Ziel, die Grundlagen für eine qualifiziertere Ausbildung aufzubauen.

\subsection{Methode der Studie und Reihenfolge der Aufgaben}

In der organisatorischen Phase der Forschung in Leipzig wurden im Mai 2018 die in Brasilien verwendeten drei Fragebögen ins Deutsche übersetzt.

Die StudentInnen wurden während der akademischen Aktivitäten (Vorlesungen, Seminare und Übungspraxis) des Sommersemesters 2018 zur Teilnahme an der Studie eingeladen. Die Aufgabe der Studie in Leipzig bestand aus der Übersetzung von einem kurzen Fachtext (mit ca. 200 Wörtern) in die Muttersprache. Es wurden Texte aus medizinischen Online-Fachzeitschriften in drei verschiedenen Ausgangssprachen (Deutsch, Englisch und Portugiesisch) ausgewählt.

Im Vergleich zur Forschung an der Bundesuniversität in Brasilien hatte die Ausweitung der Studie in Deutschland den Vorsatz, eine kontrastive Analyse der Sprachen mittels der Aufnahme von zusätzlichen Sprachpaaren durchzuführen. Als gemeinsames Ziel hatten beide Studien die Übersetzung der vorgeschlagenen Texte in die Muttersprache der ÜbersetzerInnen.

Für die Übersetzungsaufgabe wurde die Software Translog in den PCs der Computerräume heruntergeladen ${ }^{9}$. Die Software bietet den ForscherInnen die Registerprotokolle der Tastatur- und Mausbewegungen beim Übersetzen, die mit den anderen Daten der Forschung (Fragebögen und Textproduktionen beim Übersetzen) konfrontiert werden sollen ${ }^{10}$.

In den drei Tagen der Studie in Leipzig nahmen 23 StudentInnen an der Forschung teil. Die verschiedenen Sprachpaare der TeilnehmerInnen und die Benutzung oder nicht der Software werden in der folgenden Tabelle dargestellt:

\footnotetext{
9 Technische Schwierigkeiten haben die Benutzung der Software Camtasia für die Studie in Leipzig verhindert.

${ }^{10}$ Wegen technischer Probleme wurde die Software Translog nur zwei Tage bei den Experimenten benutzt. In der Systematisierung der Ergebnisse sollte diese Tatsache in Betracht gezogen werden. Die Unterschiede zwischen den beiden Gruppen (mit und ohne Software-Benutzung) können auch interessante kontrastierende Daten zeigen.
} 
Leipnitz, L.; Pickbrenner, M.B. - Das Betrachten des Übersetzungsprozesses

Tab.2: Anzahl der TeilnehmerInnen, Sprachpaare und Software-Benutzungsgruppe

\begin{tabular}{|c|c|c|}
\hline Mit Translog & Ohne Translog & Gesamte TN \\
\hline 05 EN-DE & $11 \mathrm{EN}-\mathrm{DE}$ & $16 \mathrm{EN}-\mathrm{DE}$ \\
\hline 02 DE-ES & $01 \mathrm{EN}-\mathrm{ES}$ & 03 DE/EN-ES \\
\hline $02 \mathrm{PT}-\mathrm{DE}$ & & $02 \mathrm{PT}-\mathrm{DE}$ \\
\hline $01 \mathrm{DE}-\mathrm{PT}$ & & $01 \mathrm{DE}-\mathrm{PT}$ \\
\hline $01 \mathrm{DE}-\mathrm{EN}$ & & $01 \mathrm{DE}-\mathrm{EN}$ \\
\hline $11 \mathrm{TN}$ & $12 \mathrm{TN}$ & $23 \mathrm{TN}$ \\
\hline
\end{tabular}

Die Daten in Tabelle 2 zeigen, dass insgesamt 18 StudentInnen die Ausgangstexte ins Deutsche übersetzt haben (16 vom Englischen und 2 vom Portugiesischen). Drei StudentInnen haben die Texte ins Spanische übersetzt (2 vom Deutschen und 1 vom Englischen), 1 StudentIn hat den Text vom Deutschen ins Portugiesische übersetzt und 1 StudentIn hat den Ausgangstext vom Deutschen ins Englische übersetzt. Insgesamt haben 11 TeilnehmerInnen die Software Translog benutzt und 12 StudentInnen haben die Übersetzungsaufgabe ohne Translog ausgeführt.

\subsubsection{Die Übersetzungstexte}

In der Studie in Brasilien wurden drei verschiedene kurze Texte für jede Phase der Forschung ausgewählt - eine Zusammenfassung eines Fachtextes des medizinischen Bereichs, ein journalistischer Text (kurze Nachricht aus einer Tageszeitung) und die Untertitel eines Kurzfilmes. In der Studie in Leipzig wurde nur ein Fachtext von jeder Ausgangssprache (Deutsch, Englisch oder Portugiesisch) benutzt. Der Text auf Englisch, dessen Schwierigkeitsgrad (mittelschwer) mit dem Indikator Flesch Kincaid Reading Ease (FLESCH 1974) berechnet wurde, wurde schon in der Studie in Brasilien benutzt. Die Fachtexte auf Deutsch und Portugiesisch für die Studie in Leipzig wurden nach denselben Kriterien ausgewählt. Die Sprache der Ausgangstexte, die Quelle und die Anzahl der Wörter in den Ausgangstexten werden in der folgenden Tabelle gezeigt.

Tab.3: Ausgangstexte, Quelle der Texte und Wortzahl der Texte

\begin{tabular}{|l|l|l|}
\hline $\begin{array}{l}\text { Ausgangstexte } \\
\text { Fachbereich-Medizin }\end{array}$ & Quelle - Fachzeitschriften & Wortzahl \\
\hline Deutsch & Springer Medizin & 174 \\
\hline Englisch & Annals of Behavioral Medicine & 177 \\
\hline Portugiesisch & Ciência \& Saúde Coletiva & 224 \\
\hline
\end{tabular}


Leipnitz, L.; Pickbrenner, M.B. - Das Betrachten des Übersetzungsprozesses

\subsubsection{Die Aufgaben der Übersetzerlnnen}

Die TeilnehmerInnen (TN) der Studie haben in den Computerräumen an der Universität Leipzig folgende Schritte befolgt:

1. Ausfüllung des Fragebogens zum Profil der ÜbersetzerInnen;

2. Ausfüllung des Fragebogens über die Übersetzungskonzeption;

3. Übersetzung der kurzen Texte in die Muttersprache (Deutsch, Englisch Portugiesisch oder Spanisch);

4. Ausfüllung des Fragebogens über die Übersetzungsschwierigkeiten bzw. -probleme beim Übersetzen.

Die Übersetzungsaufgaben wurden von den StudentInnen in einem durchschnittlichen Zeitraum von 1 Stunde durchgeführt. Die gesamten Aufgaben wurden in einer maximalen Laufzeit von ca. 2 Stunden gemacht. Am Ende der Aufgaben in den Computerräumen wurden alle Daten gesammelt.

\subsection{Die ersten Forschungsergebnisse}

Die Ergebnisse werden hier nach der Reihenfolge der Aufgaben in der Studie vorgestellt. Die ersten Daten stammen aus dem Fragebogen über das Profil der ÜbersetzerInnen.

\subsubsection{Das Profil der Übersetzerlnnen}

An der Studie am Institut für Angewandte Linguistik und Translatologie (IALT) in Leipzig nahmen 23 StudentInnen teil. Die erste Aufgabe der ForschungsteilnehmerInnen war das Ausfüllen des Fragebogens "Profil der ÜbersetzerInnen".

Die Daten bezüglich der Staatsangehörigkeit zeigten, dass 73,9\% der TN (17 StudentInnen) Deutsche und 26,1\% (06) Ausländer aus Brasilien, Chile, England, Spanien und aus den USA sind. 18 StudentInnen (78,3\%) sind DeutschMuttersprachlerInnen und 21,6\% haben Spanisch, Englisch oder Portugiesisch als Muttersprache.

Insgesamt studierten 82,6\% der TN (19 StudentInnen) im IALT und 17,4\% (4 TN) waren StudentInnen am Institut für Romanistik. 81\% (17 StudentInnen) der TN besuchten den Studiengang B.A. Translation (IALT) und 9,5\% (2 StudentInnen) absolvierten den 
Leipnitz, L.; Pickbrenner, M.B. - Das Betrachten des Übersetzungsprozesses

Studiengang M.A. Romanische Studien (Institut für Romanistik). Drei TN studierten M.A. Translatologie im IALT, B.A. Romanische Studien (Hispanistik/Lusitanistik und Französistik/ Lusitanistik) im Institut für Romanistik und eine StudentIn nahm damals an einem Austauchprogramm teil.

Die Mehrheit der StudentInnen 56,5\% (13 TN) befand sich im 2. Semester, ca. $30,4 \%$ besuchten das 6 . und das 7 . Semester (17,4 \% - 4 StudentInnen und 13,0\% -3 TN) und ca. $13 \%$ absolvierten das 3. oder das 4 . Semester $(8,7 \%-2 \mathrm{TN}$ und 4,6\% - $1 \mathrm{TN})$.

Die Antworten auf die Frage nach schon abgeschlossenen Studiengängen an der Universität in Leipzig, in Deutschland oder im Ausland zeigten, dass 56,5\% der ForschungsteilnehmerInnen (13 StudentInnen) keinen Studienabschluss hatten. 43,5\% der StudentInnen (10 TN) gaben an, dass sie ein Studium schon abgeschlossen hätten. Die schon abgeschlossenen Studiengänge, die erwähnt wurden, sind Germanistik, B.A. Romanische Studien, Journalismus, B.A. Romanistik (Französistik, Italianistik, Anglistik), Bachelor in Mathematik, International Business und Regenerative Energiesysteme.

52,2\% (12 StudentInnen) der TN gaben an, sie hätten noch keine Erfahrung als ÜbersetzerIn, gegen 47,8\% (11 StudentInnen) der TN, die die Frage mit „Ja“ beantworteten. Von den StudentInnen, die mit „Ja“ geantwortet haben, machten $75 \%$ (9 StudentInnen) Übersetzungen nur gelegentlich und übersetzten ausschließlich für Freunde/Bekannte oder Privatkunden. 25\% (3 StudentInnen) der TN übersetzten als Hobby, hätten jedoch noch keine berufliche Übersetzung gemacht und keine Bezahlung als ÜbersetzerIn bekommen.

Als Textsorten beim Übersetzen nannten 60\% (12 StudentInnen) der TN Wissenschafts-/Fachtexte, 35\% (7 StudentInnen) berichteten über andere Textsorten, wie populärwissenschaftliche Texte, Nachrichten, Lebensläufe, und über literarische Texte (nur 5\% - 1 StudentIn). Auf die Frage nach der Praxis-Zeit als ÜbersetzerInnen antworteten die TN 5 Monate als Minimum (2 TN - 8,7\%) und 4 Jahre als Maximum (2 TN - 8,7\%). Die Mehrheit gab an, zwischen 1 und 2,5 Jahre Erfahrung beim Übersetzen (10 TN - 43,5\%) zu besitzen. Es gab aber auch Antworten wie „nur in der Uni“, „gelegentlich“ und „,immer mal“ z.B., die für die Berechnung nicht herangezogen wurden. 73,9\% der TN (17 StudentInnen) antworteten, sie hätten Deutsch als Muttersprache, 13,0\% (3 TN) sie seien Spanisch-MuttersprachlerInnen. Es gab außerdem 
Leipnitz, L.; Pickbrenner, M.B. - Das Betrachten des Übersetzungsprozesses

noch eine Englisch-MuttersprachlerIn (4,3\%) und eine Portugiesisch-MuttersprachlerIn $(4,3 \%)$. Eine TN (4,3\%) antwortete, sie sei zweisprachig (Deutsch-Russisch).

Da die Teilnahme an der Studie freiwillig war und die Ausführung der Übersetzungsaufgabe, sowie das Ausfüllen der Fragebögen eine gewisse Zeit in Anspruch nahm, gab es keine Einschränkungen hinsichtlich der Staatsangehörigkeit, Muttersprache oder Ausbildung der TN. Die spezifischen Daten werden noch in unterschiedlichen Gruppen analysiert und nach Bewertungskriterien relativiert. Es versteht sich jedoch, dass diese Vielfalt eine breite Palette an möglichen kontrastiven Analysen bietet und das Forschungsinteresse im Bereich der Translation Studies erweckt.

\subsubsection{Die Übersetzungskonzeption der Übersetzerlnnen}

Die 27 Aussagen des Fragebogens über die Übersetzungskonzeption wurden von der PACTE-Gruppe formuliert und in der Studie über die Übersetzungskonzepte von SprachwissenschaftlerInnen und ÜbersetzerInnen im Übersetzungsprozess untersucht (PACTE 2008). Laut dieser Studie gibt es 12 dynamische und 15 statische Aussagen, darunter 5 gegensätzliche Aussagenpaare, die sich einander konzeptionell ausschließen und die Unterschiede zwischen ÜbersetzerInnen und Nicht-ÜbersetzerInnen widerspiegeln.

Mit den Daten des ausgefüllten Fragebogens über die Übersetzungskonzeption der TN in der Studie in Leipzig wurde eine Tabelle erstellt, in der die gegensätzlichen Aussagenpaare in verschiedenen Spalten nach dynamischen oder statistischen Konzepten eingeteilt wurden. Die bewerteten Antworten wurden anschließend systematisiert.

Tab.4: Teil der Tabelle mit den Antworten der ForschungsteilnehmerInnen für die gegensätzlichen Aussagenpaare

\begin{tabular}{|c|c|c|c|c|c|c|c|c|c|}
\hline $\begin{array}{c}\text { Dynamische } \\
\text { Aussage }\end{array}$ & KE & $\mathbf{E N}$ & EJ & $\mathbf{U N}$ & $\begin{array}{l}\text { Statische } \\
\text { Aussage }\end{array}$ & KE & EN & $\mathbf{E J}$ & $\mathbf{U N}$ \\
\hline $\begin{array}{l}\text { 3. Die Auftrag- } \\
\text { geberInnen der } \\
\text { Übersetzung } \\
\text { entscheiden, } \\
\text { wie die/der } \\
\text { ÜbersetzerIn } \\
\text { den Text über- } \\
\text { setzen soll. }\end{array}$ & $\begin{array}{c}\mathrm{X} \\
\% \\
(0)\end{array}$ & $\begin{array}{c}30,4 \\
\% \\
(07)\end{array}$ & $\begin{array}{c}56,5 \\
\% \\
(13)\end{array}$ & $\begin{array}{c}13,0 \\
\% \\
(03)\end{array}$ & $\begin{array}{l}\text { 24. Beim } \\
\text { Übersetzen } \\
\text { kann ich die } \\
\text { Erwartungen } \\
\text { der Zieltext- } \\
\text { leserInnen } \\
\text { ignorieren. }\end{array}$ & $\begin{array}{c}73,9 \\
\% \\
(17)\end{array}$ & $\begin{array}{c}26,1 \\
\% \\
(06)\end{array}$ & $\begin{array}{c}\mathrm{X} \\
\% \\
(0)\end{array}$ & $\begin{array}{c}\mathrm{X} \\
\% \\
(0)\end{array}$ \\
\hline
\end{tabular}


Leipnitz, L.; Pickbrenner, M.B. - Das Betrachten des Übersetzungsprozesses

Die obige Tabelle zeigt die gegensätzlichen Aussagen 3 und 24 (1. Aussagenpaar), den Prozentsatz und die Anzahl der TN für jede Antwort (KE = keinesfalls; EN = eher nein; EJ = eher ja; UN = unbedingt) nach den Grafiken des Fragebogens.

Im Folgenden werden beispielsweise die Antworten von 5 ForschungsteilnehmerInnen für die 5. gegensätzlichen Aussagenpaare gezeigt.

Tab.5: Teil der Tabelle mit den Antworten für die 5. gegensätzlichen Aussagenpaare

\begin{tabular}{|c|c|c|c|c|c|c|c|c|c|c|}
\hline $\begin{array}{c}\text { Aussagen- } \\
\text { paare }\end{array}$ & $\begin{array}{c}1 . \\
\text { Paar }\end{array}$ & $\begin{array}{c}2 . \\
\text { Paar }\end{array}$ & $\begin{array}{c}3 . \\
\text { Paar }\end{array}$ & & $\begin{array}{c}4 . \\
\text { Paar }\end{array}$ & & $\begin{array}{c}5 . \\
\text { Paar }\end{array}$ & \\
\hline TN & 3 & 24 & 10 & 4 & 23 & 11 & 14 & 5 & 27 & 16 \\
\hline & dyn & sta & dyn & sta & dyn & sta & dyn & sta & dyn & sta \\
\hline 1 & EJ & KE & EJ & EJ & EJ & EN & KE & EN & EN & EJ \\
\hline 2 & UN & KE & EJ & EJ & EJ & EJ & KE & EN & UN & EN \\
\hline 3 & UN & EN & UN & EJ & UN & KE & EJ & EN & EJ & EJ \\
\hline 4 & EJ & KE & EN & EN & EJ & EN & EJ & EN & UN & EJ \\
\hline 5 & EJ & KE & EJ & EJ & EJ & EJ & KE & KE & EN & EJ \\
\hline
\end{tabular}

Um die Übersetzungskonzepte der TN auszuwerten, wurden Punkte für jede Antwort gegeben. Die Punkte entsprechen einem dynamischeren oder statischeren Übersetzungskonzept der ÜbersetzerInnen und wurden nach dem folgenden Auswertungssystem aufsummiert.

Tab.6: Auswertungssystem für die Antworten der 5.Aussagenpaare

\begin{tabular}{|c|c|c|c|}
\hline $\begin{array}{c}\text { Auswertung der } \\
\text { dynamischen } \\
\text { Aussagen }\end{array}$ & $\begin{array}{c}\text { Auswertung der } \\
\text { statischen } \\
\text { Aussagen }\end{array}$ & \\
\hline Unbedingt & +1 & Unbedingt & -1 \\
\hline Eher ja & $+0,5$ & Eher ja & $-0,5$ \\
\hline Ehe nein & $-0,5$ & Eher nein & $+0,5$ \\
\hline Keinesfalls & -1 & Keinesfalls & +1 \\
\hline
\end{tabular}

Nach diesem System, wie oben gezeigt, wird eine Antwort ,unbedingt” für eine dynamische Aussage durch einen +1 Punkt gekennzeichnet. Das würde zeigen, dass die ÜbersetzerIn ein dynamisches Übersetzungskonzept hat. Wenn die ÜbersetzerIn z.B. „keinesfalls“ für eine dynamische Aussage angibt, wird dies durch einen -1 Punkt gekennzeichnet, weil es keine adequäte Antwort gegenüber einem dynamischen Konzept beim Übersetzen darstellt. Genau das Gegenteil ist für die Antworten zu statischen 
Leipnitz, L.; Pickbrenner, M.B. - Das Betrachten des Übersetzungsprozesses

Aussagen der Fall: eine „keinesfalls“ Antwort wird durch einen +1 Punkt gekennzeichnet, weil sie bedeutet, dass das Übersetzungskonzept einen Dynamismus ausdrückt.

Anhand der Summe der Punkte wurden die individuellen Übersetzungskonzepte der ForschungsteilnehmerInnen ausgewertet und der Konsistenz-Index aller TN systematisiert. Dieser Konsistenz-Index wird aber noch mit den anderen Daten der Forschung kontrastiert.

Im nachfolgenden Schritt der Forschung wurde der Fragebogen über die Schwierigkeiten und Probleme beim Übersetzen ausgewertet.

\subsubsection{Die Schwierigkeiten und Probleme beim Übersetzen}

Um die Textproduktion der ÜbersetzerInnen auszuwerten, mussten die Ergebnisse des Fragebogens "Übersetzungsschwierigkeiten und Übersetzungsprobleme" vorher erhoben werden. Nach der PACTE-Gruppe (2014) und nach der Studie in Brasilien (LIPARINI CAMPOS, LEIPNITZ, BRAGA 2017) wurden die rich-points in den Ausgangstexten identifiziert, um die Auswertungskriterien für die Zieltexte zu etablieren.

Die rich-points entsprechen den Schwerpunkten des Ausgangstextes, die vorher ausgewählt wurden und Übersetzungsprobleme verursachen könnten. Die Qualität des Zieltextes soll nach diesen Kriterien gemessen werden. Zu diesem Zweck wurden die Schwierigkeiten und Probleme der ÜbersetzerInnen systematisiert, um sie im Laufe der Forschung mit den Übersetzungslösungen zu kontrastieren.

Die erste Frage des Fragebogens, über den Schwierigkeitsgrad des Ausgangstextes, enthielt eine Graduierung der Skala von „,zu einfach” (1) bis ,zu schwer” (10). Zu den Schwerpunkten beim Übersetzen wurden geschlossene und offene Fragen gestellt. Die zweite Frage über die Merkmale des Textes, die die Schwierigkeiten beim Übersetzen behandelt, ist z.B. eine offene Frage; in der geschlossenen Frage Nummer 3 wiederum werden einige Schwerpunkte beim Übersetzen abgefragt: Wortschatz, sprachliche Unterschiede, Terminologie, Fachsprache, kulturspezifische Unterschiede, außersprachliche Kenntnisse, die besseren Forschungsquellen kennen/finden/benutzen, die effektivere Übersetzungsmethode kennen/finden/benutzen, eine effektivere Übersetzungsstrategie kennen/finden/benutzen. Der vierte Fragekomplex des Fragebogens enthält geschlossene und offene Fragen. Die TN sollten anhand der drei schwierigsten Textteile ihre Schwerpunkte beim Übersetzen darstellen und Kommentare 
Leipnitz, L.; Pickbrenner, M.B. - Das Betrachten des Übersetzungsprozesses

zu den Schwierigkeiten und den gefundenen Lösungen geben. Die Antworten wurden systematisiert und werden später mit den anderen Daten der Forschung und mit der Textproduktion der ÜbersetzerInnen kontrastiert, um den Übersetzungsprozess der TN auszuwerten.

\subsubsection{Die Auswertung der Textproduktion}

Laut der Studie der PACTE-Gruppe (2003) ist es möglich, mit empirischen Methoden die spezifischen Subkompetenzen der ÜbersetzerInnen zu untersuchen. Es wird außerdem behauptet, dass die Qualität des Zieltextes ein notwendiger Faktor in den Studien über Übersetzungskompetenz ist. Diese notwendige Qualität ist aber nur ein Indikator, dessen Daten mit anderen Indikatoren der Subkompetenzen der ÜbersetzerIn transversal korreliert werden müssen (PACTE 2014).

Ausgehend von der Annahme, dass die Qualität des Endproduktes bzw. des Übersetzungstextes ein wichtiger Faktor in den Studien über Übersetzungskompetenz ist, stellt sich die Frage, wie die ForscherInnen die Übersetzungsqualität messen oder bewerten können.

Mit der Zielsetzung, eine Antwort auf diese Frage zu finden, arbeiten viele WissenschaftlerInnen mit verschiedenen Forschungsperspektiven (vgl. u. a. LIPARINI Campos 2008, 2010; LoRenzo 2002; Rothe Neves 2002; Braga 2012; PACTE 2014; CAStillo 2015).

Die Forschung in João Pessoa untersuchte den Fortschritt der StudentInnen des Studiengangs Übersetzungswissenschaft im Verlauf von drei Jahren (LIPARINI CAMPOS, LEIPNITZ 2017; LiPARINI CAMPOS, LEIPNITZ, BRAGA 2017). Die Textproduktionen der künftigen ÜbersetzerInnen wurden von DozentInnen des Studiengangs mit Unterstützung der Parameter von PACTE (2011b) und Braga (2012) und vom TQA Tool (COLINA 2008) ausgewertet. Das Tool betrachtet die Übersetzungsaufgabe, die Zielgruppe und die kommunikative Funktion der Übersetzung. Auf dieser Linie sind die Kriterien, die Ziele und das Verhalten der TN für die Auswertung eines Übersetzungstextes von dem Kontext der Übersetzung abhängig, d.h., ob die Aufgabe in der Ausbildung oder in der beruflichen Praxis (ALBIR 2007) angewandt wird. In der Übersetzungsausbildung soll der Fokus nicht nur auf dem Zieltext, auf dem Übersetzungsprodukt, sondern auch auf dem 
Leipnitz, L.; Pickbrenner, M.B. - Das Betrachten des Übersetzungsprozesses

Übersetzungsprozess und auf dem Fortschritt des Lernprozesses der ÜbersetzerInnen liegen.

Gemäß den Methoden von PACTE (2011b) und den Auswertungsparametern in João Pessoa (LIPARINI CAMPOS, LEIPNITZ, BRAGA 2017) wurden die Ausgangstexte und die Zieltexte als bestimmte Textsorte identifiziert. Das bedeutet, dass die Textfunktion, die kommunikative Funktion des Textes und die Zielgruppe des Textes in den Auswertungen betrachtet wurden. Während in João Pessoa die Daten für drei verschiedene Textsorten (Fachtext, Zeitungsnachricht und Kurzfilm-Untertitel) etabliert wurden, wurden die Kriterien in der Studie in Leipzig nur für den Fachtext übernommen.

Nach diesen Kriterien sollten die GutachterInnen die Textproduktionen der StudentInnen aus vier verschiedenen Bestandteilen (Zielsprache, textuelle und funktionelle Adäquatheit der Zieltexte, nicht fachspezifischer Inhalt und fachspezifischer Inhalt) auswerten. Jedem Bestandteil werden vier verschiedene Kategorien zugeschrieben. Zu jeder Kategorie gehören bestimmte Auswertungspunkte, deren Wert zwischen 1 und 4 bewertet wurde. Die Skala zwischen 1 bis 4 entspricht der Relevanz der Kategorie in der Bewertung des Übersetzungstextes.

Tab.7: Die Punkte für jeden Auswertungsbestandteil nach der Textsorte „Fachtext“ (LIPARINI CAMPOS, LEIPNITZ, BRAGA 2017)

\begin{tabular}{|c|c|c|}
\hline Textsorte & Bestandteil & Punkte \\
\hline Fachtext & Zielsprache & 1 \\
\cline { 2 - 3 } & Textuelle und funktionelle Adäquatheit & 4 \\
\cline { 2 - 3 } & Nicht-fachspezifischer Inhalt & 2 \\
\cline { 2 - 3 } & Fachspezifischer Inhalt & 3 \\
\hline
\end{tabular}

Laut der Studie in João Pessoa (LIPARINI CAMPOS, LEIPNITZ, BRAGA 2017) ist der Bestandteil „Textuelle und funktionelle Adäquatheit"“ das wichtigste Auswertungskriterium (4 Punkte), da diese Kompetenz impliziert, dass die Übersetzungstexte den kommunikativen Zweck im Kontext, die Bedürfnisse der AuftraggeberIn und des Zielpublikums erfüllen müssen, d.h. dass die Übersetzungstexte auf dem Übersetzungsmarkt akzeptiert werden sollen. Der fachspezifische Inhalt entspricht 3 Punkten, weil die Kenntnis und der geeignete Gebrauch der Fachwörter für eine erfolgreiche Fachkommunikation bzw. für den Informationsaustausch der wissenschaftlichen Studien verantwortlich sind. Der Bestandteil „nicht fachspezifischer 
Leipnitz, L.; Pickbrenner, M.B. - Das Betrachten des Übersetzungsprozesses

Inhalt" wiederum ergänzt den fachspezifischen Inhalt und wird mit 2 Punkten bewertet. Der Bestandteil „Zielsprache“ wird mit 1 Punkt bewertet.

Die Bewertungsskala wird aber nur von der ForscherIn der Studie benutzt, um die Punkte der Bestandteile zu sammeln und die Abschlussnote für die Textproduktion bzw. für die Übersetzungstexte zu geben. Die Auswertung der GutachterInnen wurde nur anhand der 4 Bestandteile und Kategorien ausgeführt. Im Hinblick auf die Notwendigkeit von Sprachkenntnissen in der Ausgangssprache zur Auswertung der 3. und 4. Bestandteile wurde noch eine Kategorie für die Auswertung der Texte in der Studie in Leipzig erstellt. Nach jedem Bestandteil gibt es auch ein Feld für Kommentare der GutachterInnen, in dem sie ihre Auswahl begründen sollen.

Die vier Bestandteile und die entsprechenden Kategorien werden in der folgenden Tabelle gezeigt:

Tab.8: Auswertungskategorien für jeden Bestandteil

(vgl. LIPARINI CAMPOS, LEIPNITZ, BRAGA 2017)

\begin{tabular}{|c|c|}
\hline Bestandteile & Kategorien \\
\hline \multirow{4}{*}{ 1) Zielsprache } & Der Text ist unverständlich. \\
\hline & $\begin{array}{l}\text { Der Text ist schwer zu verstehen und hat Strukturen und Elemente } \\
\text { des Ausgangstextes, die nicht typisch in der Zielsprache sind. }\end{array}$ \\
\hline & $\begin{array}{l}\text { Der Text ist lesbar, hat aber einige ungewöhnliche Ausdrücke, } \\
\text { die sonderbar klingen. }\end{array}$ \\
\hline & $\begin{array}{c}\text { Der Text ist fast genauso wie ein Text, der in der Zielsprache } \\
\text { geschrieben wurde. }\end{array}$ \\
\hline \multirow{4}{*}{$\begin{array}{l}\text { 2) Textuelle und } \\
\text { funktionelle } \\
\text { Adäquatheit }\end{array}$} & $\begin{array}{l}\text { Der Text betrachtet nicht die kommunikative Situation, die } \\
\text { Übersetzungsfunktion und die vorgeschlagenen } \\
\text { ZieltextleserInnen. Es ist unmöglich den Text zu verbessern. }\end{array}$ \\
\hline & $\begin{array}{l}\text { Der Text betrachtet teilweise die kommunikative Situation, die } \\
\text { Übersetzungsfunktion und die ZieltextleserInnen und benötigt } \\
\text { große Anstrengung, um verbessert zu werden. }\end{array}$ \\
\hline & $\begin{array}{c}\text { Der Text kommt der kommunikativen Situation, der } \\
\text { Übersetzungsfunktion und den ZieltextleserInnen des } \\
\text { Ausgangstextes nah und kann ohne weitere Bearbeitungen } \\
\text { verbessert werden. }\end{array}$ \\
\hline & $\begin{array}{l}\text { Der Text betrachtet die kommunikative Situation, die } \\
\text { Übersetzungsfunktion und die ZieltextleserInnen und benötigt } \\
\text { fast keine Textüberprüfung. }\end{array}$ \\
\hline \multirow{3}{*}{$\begin{array}{l}\text { 3) Nicht- } \\
\text { fachspezifischer } \\
\text { Inhalt }\end{array}$} & $\begin{array}{l}\text { Der Text enthält ungerechtfertigte Abweichungen im Vergleich } \\
\text { mit dem Ausgangstext (Der Zieltext ist fehlerhaft). }\end{array}$ \\
\hline & $\begin{array}{l}\text { Der Text enthält einige inhaltliche Missverständnisse im } \\
\text { Vergleich mit dem Ausgangstext. }\end{array}$ \\
\hline & Der Text enthält geringfügige Änderungen der textuellen \\
\hline
\end{tabular}

Pandaemonium, São Paulo, v. 23, n. 40, mai.-ago. 2020, p. 140-165 
Leipnitz, L.; Pickbrenner, M.B. - Das Betrachten des Übersetzungsprozesses

\begin{tabular}{|c|c|}
\hline \multirow{2}{*}{\begin{tabular}{|c|} 
Bedeutung und einige Erweiterungen und Auslassungen im \\
Vergleich mit dem Ausgangstext.
\end{tabular}} \\
\cline { 2 - 2 } $\begin{array}{c}\text { 4) Fach- } \\
\text { spezifischer } \\
\text { Inhalt }\end{array}$ & $\begin{array}{c}\text { Der Text spiegelt mit Genauigkeit den Inhalt des Ausgangstextes } \\
\text { wider. }\end{array}$ \\
\hline \multirow{4}{*}{$\begin{array}{c}\text { Eer Textinhalt zeigt, dass die TextproduzentIn die Terminologie } \\
\text { des Ausgangstextes nicht kennt und keine adäquaten Kenntnisse } \\
\text { über den fachspezifischen Inhalt hat. }\end{array}$} \\
\cline { 2 - 2 } & $\begin{array}{c}\text { Der Text hat viele und gravierende Fehler in Bezug auf die } \\
\text { Terminologie und den fachspezifischen Inhalt. }\end{array}$ \\
\cline { 2 - 2 } & $\begin{array}{c}\text { Der Text hat einige terminologische Fehler, aber der } \\
\text { fachspezifische Inhalt wurde nicht stark davon beeinflusst. }\end{array}$ \\
\cline { 2 - 2 } & $\begin{array}{c}\text { Der Text ist präzise und adäquat in Bezug auf den } \\
\text { fachspezifischen Inhalt. }\end{array}$ \\
\hline & Es besteht keine Möglichkeit, eine Auswertung zu erstellen. \\
\hline
\end{tabular}

Die GutachterInnen sollten keine grammatikalische und detaillierte Auswertung durchführen, sondern die gesamte Qualität des Zieltextes als übersetzter Text aus dem fachspezifischen Bereich auswerten. Die Formulare entsprechen den vier Kategorien der Bestandteile als geschlossene Fragen. Am Ende sollte noch eine Gesamtbewertung der Übersetzung eingetragen werden. Die GutachterInnen konnten den Übersetzungstext nach 4 verschiedenen Kriterien kategorisieren: (1) der Text ist adäquat, (2) muss leicht korrigiert werden, (3) muss gründlich korrigiert werden oder (4) muss umgeschrieben werden. Zum Schluss wurde noch ein Kommentar zum Text geschrieben.

Die Texte zur Auswertung wurden den Zielsprachen zufolge in 4 Gruppen geteilt: Gruppe 1 - Englisch-Deutsch, Gruppe 2 - Deutsch-Spanisch/Englisch-Spanisch, Gruppe 3 - Portugiesisch-Deutsch und Gruppe 4 - Deutsch-Portugiesisch und DeutschSpanisch $^{11}$. Die erste Auswertungsgruppe war die Gruppe 1 - Sprachpaar EnglischDeutsch, die insgesamt 16 TN bzw. Zieltexte enthielt. Die TN in dieser Gruppe haben den Ausgangstext vom Englischen ins Deutsche (ihre Muttersprache) übersetzt.

\footnotetext{
${ }^{11}$ Diese Gruppe wurde mit zwei verschiedenen Zielsprachen gebildet, weil nur eine TeilnehmerIn für jedes Sprachpaar an der Studie teilnahm. Die Gruppen 2, 3 und 4 gelten als Vergleich-Parameter in der Studie. Die Anzahl der TeilnehmerInnen dieser Gruppe ist zu niedrig, um die isolierten Daten zu betrachten, aber die Daten der unterschiedlichen Sprachen in Kontrast können auch interessante Ergebnisse darstellen. Die verschiedenen Sprachpaare beim Übersetzen hatten, wie schon erwähnt, als gemeinsames Kriterium, den Ausgangstext in die jeweilige Muttersprache zu übersetzen. Die Gruppe 1 ist die relevanteste Gruppe in der Studie wegen der Anzahl der TeilnehmerInnen, die denselben Ausgangstext der Studie in Brasilien vom Englischen ins Deutsche übersetzten.
}

Pandaemonium, São Paulo, v. 23, n. 40, mai.-ago. 2020, p. 140-165 
Leipnitz, L.; Pickbrenner, M.B. - Das Betrachten des Übersetzungsprozesses

Vier GutachterInnen haben die Zieltexte der Gruppe 1 ausgewertet: zwei Germanistik-DozentInnen und zwei ÜbersetzerInnen mit nachgewiesener Kompetenz in der deutschen Sprache.

Mit den Auswertungsergebnissen der Textproduktion wurde eine Tabelle mit den individuellen Punkten der TN organisiert und eine kontrastive Analyse durchgeführt.

Tab.9: Beispiel der Auswertungsergebnisse der Textproduktion einer Studie-TN

\begin{tabular}{|c|c|c|c|c|}
\hline Bestandteile & Kategorien & Note & Punkte & $\begin{array}{l}\text { Gut- } \\
\text { achter } \\
\text { In }\end{array}$ \\
\hline \multirow{4}{*}{$\begin{array}{l}\text { 2) Textuelle und } \\
\text { funktionelle } \\
\text { Adäquatheit (4) }\end{array}$} & $\begin{array}{l}\text { Der Text betrachtet nicht die kommunikative } \\
\text { Situation, die Übersetzungsfunktion und die } \\
\text { vorgeschlagenen ZieltextleserInnen. Es ist } \\
\text { unmöglich denText zu verbessern. }\end{array}$ & 1 & & \\
\hline & $\begin{array}{l}\text { Der Text betrachtet teilweise die kommunikative } \\
\text { Situation, die Übersetzungsfunktion und die } \\
\text { ZieltextleserInnen und benötigt große } \\
\text { Anstrengung, um verbessert zu werden. }\end{array}$ & 2 & $\begin{array}{l}8 \\
8\end{array}$ & $\begin{array}{l}3 \mathrm{D} \\
4 \mathrm{D}\end{array}$ \\
\hline & $\begin{array}{c}\text { Der Text kommt der kommunikativen Situation, } \\
\text { der Übersetzungsfunktion und den } \\
\text { ZieltextleserInnen des Ausgangstextes nah und } \\
\text { kann ohne weitere Bearbeitungen verbessert } \\
\text { werden. }\end{array}$ & 3 & $\begin{array}{l}12 \\
12\end{array}$ & $\begin{array}{l}1 \mathrm{D} \\
2 \mathrm{D}\end{array}$ \\
\hline & $\begin{array}{c}\text { Der Text betrachtet die kommunikative Situation, } \\
\text { die Übersetzungsfunktion und die } \\
\text { ZieltextleserInnen und benötigt fast keine } \\
\text { Textüberprüfung. }\end{array}$ & 4 & & \\
\hline
\end{tabular}

Die obengenannte Tabelle zeigt in der ersten Spalte die Punktwertung für den Bestandteil „Textuelle und funktionelle Adäquatheit“, in der dritten die Note der GutachterInnen nach den Antworten des Auswertungsformulars, dann die individuellen Punkte der TN für den Bestandteil (4. Spalte) und die GutachterIn-Code (5. Spalte). Nach den Auswertungskriterien kann der Bestandteil „Textuelle und funktionelle Adäquatheit““ maximal 4 Punkte bekommen. Nach der Auswertung der GutachterIn 2D z.B. sollte die TN nun 3 Punkte bekommen. Das Ergebnis der Punktwertung 4 Punkte x 3 Punkte beträgt insgesamt 12 Punkte für den Bestandteil „Textuelle und funktionelle Adäquatheit“" dieser TN.

Zum Schluss wurde die Summe der Punkte von jeder GutachterIn durch 10 geteilt, um die Noten der Textproduktion der $\mathrm{TN}$ zu etablieren. Die Noten entsprechen verschiedenen Stellungnahmen. 
Leipnitz, L.; Pickbrenner, M.B. - Das Betrachten des Übersetzungsprozesses

Tab.10: Beispiel der Auswertungsergebnisse mit Punkten, Noten und Stellungnahme der GutachterInnen

\begin{tabular}{|c|c|c|c|c|}
\hline $\begin{array}{c}\text { Gesamte } \\
\text { Punkte }\end{array}$ & GutachterIn & & Note & Stellungnahme \\
\hline 27 & 1D & $27 / 10$ & 2,7 & $\begin{array}{c}\text { Der Text muss leicht korrigiert } \\
\text { werden. }\end{array}$ \\
32 & 2D & $32 / 10$ & 3,2 & $\begin{array}{c}\text { Der Text muss leicht korrigiert } \\
\text { werden. }\end{array}$ \\
\hline
\end{tabular}

Die Stellungnahme folgen der Auswertungskriterien der Studie in Brasilien, die in der folgenden Tabelle gezeigt werden:

Tab.11: Auswertungskriterien nach der Studie in Brasilien (LIPARINI CAMPOS, BRAGA, LEIPNITZ 2017)

\begin{tabular}{|c|c|}
\hline Auswertungskriterien & Noten \\
\hline Der übersetzte Text muss umgeschrieben werden. & 1,00 bis 1,75 \\
\hline $\begin{array}{c}\text { Der übersetzte Text muss gründlich korrigiert } \\
\text { werden. }\end{array}$ & 1,76 bis 2,50 \\
\hline Der übersetzte Text muss leicht korrigiert werden. & 2,51 bis 3,25 \\
\hline Der übersetzte Text ist adäquat. & 3,26 bis 4,00 \\
\hline
\end{tabular}

Die Auswertungen der Textproduktion der ÜbersetzerInnen, die Analyse der Daten des Fragebogens über die Schwierigkeiten und Probleme beim Übersetzen und der Daten der Translog Protokolle und Graphiken sind noch nicht abgeschlossen. Eine Kontrastierung mit der Forschung in Brasilien ermöglicht es aber, über die primären Ergebnisse der Studie zu berichten, sowie die zukünftigen Schritte der Datenanalyse und Forschungsperspektiven vorzustellen.

\section{Primäre Ergebnisse der Konstrastierung beider Forschungsprojekte}

Es gibt Daten der Studie in Leipzig, die noch ausgewertet werden müssen. Die Protokolle und Daten der Software Translog z.B. wurden noch nicht analysiert. Wie schon erwähnt, machte wegen technischer Probleme nur ein Teil der TN-Gruppe (11 StudentInnen) die Übersetzungsaufgabe mit der Software im Computerraum. Mit den Daten der Software 
Leipnitz, L.; Pickbrenner, M.B. - Das Betrachten des Übersetzungsprozesses

ist es möglich, Informationen über die Textlöschungen, die Dauer der Textproduktion, die Schritte pro Minute beim Übersetzen u.a. zu analysieren. Mit Hilfe der Daten über die Dauer der Textproduktion der TN wurden z.B. unterschiedliche Zeiträume beobachtet: eine Mindestdauer von ca. 28 Minuten und eine Höchstdauer von 1 Stunde und 27 Minuten. Die Betrachtung solcher Informationen in Triangulation mit anderen Daten bzw. mit der Texproduktion und der Übersetzungskonzeption der StudentInnen können zu interessanten Ergebnissen führen.

Was die Profile der ÜbersetzerInnen angeht, gibt es große Unterschiede zwischen den beiden StudentInnengruppen. Die StudentInnen in Deutschland bestätigten, Sprachkenntnisse in mehr als zwei Fremdsprachen und verschiedene Auslandserfahrungen zu haben. Die BrasilianerInnen wiederum berichteten, keine Erfahrung im Ausland zu haben und normalerweise auch keine guten Sprachkenntnisse zu besitzen. Diese Unterschiede können vielleicht auf die geographische Lage Deutschlands zurückgeführt werden, da es für StudentInnen, die sich in Deutschland befinden, z.B. einfacher sein kann, in einem anderem Land zu leben bzw. zu studieren oder eine Fremdsprache im Ausland zu lernen als für StudentInnen, die in Brasilien leben.

Wenn die Übersetzungskonzepte der ÜbersetzerInnen beider Studien miteinander verglichen werden, gibt es zwar unterschiedliche Ergebnisse, aber insgesamt konnten in Bezug auf die Kenntnisse der zukünftigen ÜbersetzerInnen in Brasilien und in Deutschland mehr Ähnlichkeiten als Unterschiede identifiziert werden.

Der durchschnittliche Index des Übersetzungskonzeptes der StudentInnen in der Studie in João Pessoa lag beispielsweise bei 0,05 in der ersten Phase der Forschung (2014) und bei 0,4 in der zweiten Phase (2015) (LIPARINI CAMPOS, LEIPNITZ 2017). In der gesamten TN-Gruppe (23 StudentInnen) in Leipzig lag der durchschnittliche Index des Übersetzungskonzeptes bei 0,3 und in der 1. Auswertungsgruppe (16 StudentInnen, die vom Englischen ins Deutsche übersetzten) lag er bei 0,4. Bei einer komparativen Analyse beider Studien signalisiert derselbe Index interessante Forschungsperspektiven. Solche Daten sollen auch zusätzlich mit den Ergebnissen anderer Analysemethoden kontrastiert werden.

In Bezug auf die Evaluierungen der Textproduktionen der TN wurde bis zu diesem Zeitpunkt die Analyse der Punktzahl, die nach den Auswertungskriterien der Forschung an der Bundesuniversität in João Pessoa bestimmt wurden, durchgeführt. Die übersetzten Texte befinden sich sozusagen in einer Evaluierungsphase. Anhand der aus der Punktzahl 
Leipnitz, L.; Pickbrenner, M.B. - Das Betrachten des Übersetzungsprozesses

resultierenden Ergebnisse wird es z.B. möglich sein, einen ersten Vergleich mit den Resultaten des dynamischen Indexes zu den Übersetzungskenntnissen der TN aufzustellen. Laut dieser Indexe wurden Parameter für eine substanzielle oder oberflächliche Überarbeitung der übersetzten Texte festgelegt.

Die Ergebnisse der ersten Auswertungsphase der Textproduktion der TN werden mit den Ergebnissen von Bemerkungen, die in der (schon angefangenen) zweiten Phase durchgeführt werden, kontrastiert. Diese Informationen hinsichtlich der Textproduktion der Gruppe, die vom Englischen ins Deutsche übersetzt hat, brachten interessante Erkenntnisse, die in der Fortsetzung dieser Forschung ausführlicher betrachtet werden. Die ersten Ergebnisse weisen dennoch auf eine noch inkonsistente Übersetzungskonzeption der deutschen StudentInnen hin.

Es wurden Textteile in der Zielsprache betrachtet, die aufgrund einer zu starken Orientierung am Ausgangstext fragmentiert aussehen und kein fließendes Lesen ermöglichen. In dieser Hinsicht wurden textuelle Strukturen im Deutschen identifiziert, die Sprachstrukturen des Englischen einfach wiederholen. Auf diese Weise wurden Zieltexte mit wörtlich übersetzten Textteilen erzeugt und pragmatisch-kommunikative Dimensionen der Textsorte „wissenschaftlicher Artikel des medizinischen Bereichs“ nicht berücksichtigt. Weil sie zu sehr der Textstruktur des Ausgangstextes verhaftet sind, wurde auch identifiziert, dass die Reihenfolge der verbnominalen Wortverbindungen nicht beachtet wurde. Als Beispiel dafür werden in der folgenden Tabelle zwei Textausschnitte aus dem Ausgangstext (AT) im Englischen und die entsprechende Übersetzungswahl von drei deutschen StudentInnen für den Zieltext (ZT) dargestellt. Es muss hier betont werden, dass sonstige Inkonsistenzen bezüglich der textuellen Produktion im Deutschen nicht berücksichtigt wurden, da der Schwerpunkt der Analyse auf die Textstruktur gelegt wurde. Spezifische Aspekte zu diesem Thema werden, wie schon erwähnt, in einer künftigen Arbeit behandelt. 
Leipnitz, L.; Pickbrenner, M.B. - Das Betrachten des Übersetzungsprozesses

Tab. 12: Textausschnitte aus dem Ausgangstext und die Textproduktion in der deutschen Sprache

\begin{tabular}{|l|l|}
\hline AT & $\begin{array}{l}\text { Social isolation has been linked to a variety of adverse health outcomes, including } \\
\text { cardiovascular disease. }\end{array}$ \\
\hline ZT8 & $\begin{array}{l}\text { Soziale Isolation wird in Zusammenhang mit diversen gesundheitsschädlichen } \\
\text { Folgen gebracht, kardiovaskuläre Erkrankungen inbegriffen. }\end{array}$ \\
\hline ZT13 & $\begin{array}{l}\text { Soziale Isolation ist verbunden mit einer Vielzahl von Gesundheitsproblemen, } \\
\text { einschließlich der Herzkrankheit. }\end{array}$ \\
\hline AT & $\begin{array}{l}\text { Researchers have attributed this association to the feelings of loneliness that } \\
\text { accompany social isolation, but they have rarely assessed loneliness directly. }\end{array}$ \\
\hline ZT9 & $\begin{array}{l}\text { Wissenschaftler haben dies in Verbindung gebracht mit Gefühlen der Einsamkeit, } \\
\text { die soziale Isolierung begleiten. Sie haben aber selten Einsamkeit direkt bewertet. }\end{array}$ \\
\hline
\end{tabular}

Anhand der obigen Beispiele lässt sich die „starke Bindung“ der Zielsprache zur Struktur des Englischen und die daraus resultierende Nichtberücksichtigung der grammatischen Strukturen des Deutschen erkennen, wie in Studien von Baker (1994), Vasconcellos (1997), Dragsted (2004) und Alves (2005), die Vergleiche zwischen der Textproduktion von ÜbersetzerstudentInnen und professionellen ÜbersetzerInnen angestellt haben, festgestellt wurde.

Erfahrene ÜbersetzerInnen identifizieren, laut Ergebnissen der oben genannten Studien, pragmatisch-kommunikative Dimensionen des Ausgangstextes und produzieren größere Übersetzungseinheiten (mehr als 10 Wörter pro Einheit) in kürzerer Zeit, indem Satzkomplexe und satzübergreifende Strukturen verarbeitet werden.

Beide Aspekte, bzw. die Produktion von kürzeren Übersetzungseinheiten und die Beibehaltung derselben Strukturen des Ausgangstextes, wie in der Tabelle gezeigt, wurden sowohl bei der Textproduktion der deutschen als auch bei den brasilianischen StudentInnen bemerkt, was in direktem Zusammenhang steht mit einer mangelnden Übersetzungserfahrung bezüglich der Übersetzung von fachspezifischen Textsorten seitens der genannten ForschungsteilnehmerInnen. Daher sollte dieses Thema als Lehrobjekt behandelt werden und eine wichtige Rolle bei der beruflichen Bildung von ÜbersetzerInnen spielen. Ebenso wichtig ist hierbei die Durchführung von neuen longitudinalen Forschungen, um die Entwicklung der Übersetzungskompetenz der deutschen StudentInnen so betrachten zu können, wie es bereits in der Studie an der Universität in Brasilien vorgenommen wurde.

Weitere Aspekte dieser Forschung, die aktuell noch analysiert werden, müssen ebenfalls näher in Betracht gezogen werden, bevor man zu umfassenden, auch didaktisch 
Leipnitz, L.; Pickbrenner, M.B. - Das Betrachten des Übersetzungsprozesses

relevanten Ergebnissen gelangen kann ${ }^{12}$. In diesem Sinne wird die vorhandene Forschung unter folgenden Punkten weitergeführt mit dem Ziel, noch diferenziertere Ergebnisse zur Kontrastierung mit schon dargestellten Daten vorzulegen:

- 1. Abschluss der Auswertungen der Textproduktion;

- 2. Analyse der Auswertungen und Kommentare der GutachterInnen;

- 3. Analyse der Translog-Protokolle und Statistik-Daten;

- 4. Komparative Analyse der Ergebnisse mit verschiedenen Sprachpaaren;

- 5. Triangulation der Ergebnisse der Studie in Leipzig;

- 6. Vergleich mit den Ergebnissen der Studie in Brasilien.

Die aus den neuen Analysen resultierenden Ergebnisse können dabei hilfreich sein, die Übersetzungsdidaktik im Bereich der Ausbildung von ÜbersetzerInnen zu entwickeln und die Beantwortung der folgenden Forschungsfrage zu ermöglichen:

Sollen die Lehr-Methoden in Übersetzungsstudiengängen universell sein oder gibt es eine universelle und eine sprach- und kulturspezifische Übersetzungsdidaktik, um eine effektivere Entwicklung der Übersetzungskompetenz zu gewährleisten?

\section{Literaturverzeichnis}

ALVES, F. Esforço cognitivo e efeito contextual em tradução: relevância no desempenho de tradutores novatos e expertos. Linguagem em (Dis)curso, Tubarão, v. 5, 2005, p. 11-31.

BAKER, M. In other words: a coursebook on translation. London; New York: Routledge, 1994.

BRAGA, C.N.O. O texto traduzido sob a perspectiva do avaliador: um estudo exploratório. Tese de Doutorado em Linguística Aplicada. Faculdade de Letras da Universidade Federal de Minas Gerais/Poslin, Belo Horizonte, 2012.

CASTILlO, L.M. Acquisition of translation competence and translation acceptability: an experimental study. Translation \& Interpreting, v. 7, n. 1, 2015, p. 72-85.

CoLINA, S. Translation Quality Evaluation: empirical evidence for a functionalist approach. The Translator, London, v. 14, n. 1, 2008, p. 97-134. Disponível em: https://doi.org/10.1080/13556509.2008.10799251 (Acesso: 20/11/2019)

DRAGSTED, B. Segmentation in translation and translation memory systems. (Unpublished PhD thesis). Copenhagen: Copenhagen Business School, 2004.

FLESCH, R. The art of readable writing. New York, NY: Harper, 1974.

\footnotetext{
${ }^{12}$ Seit Juli 2019 wird ein Projekt zur Einführung in die Forschungspraxis an der Universität in João Pessoa entwickelt. Die TeilnehmerInnen des Projektes, ÜbersetzungsstudentInnen, die von ForscherInnen und DozentInnen beraten werden, wurden dafür engagiert, die noch fehlenden Phasen der Forschung durchzuführen und abzuschließen. Die Ergebnisse dieser Arbeit werden in einer nächsten Publikation präsentiert.
}

Pandaemonium, São Paulo, v. 23, n. 40, mai.-ago. 2020, p. 140-165 
Leipnitz, L.; Pickbrenner, M.B. - Das Betrachten des Übersetzungsprozesses

GONÇALVES, J.L.V.R. O Desenvolvimento da Competência do Tradutor: investigando o processo através de um estudo exploratório-experimental. Tese de Doutorado em Linguística Aplicada. Faculdade de Letras da UFMG/Poslin, Belo Horizonte, 2003.

GONÇALVES, J.L.V.R. O desenvolvimento da competência do tradutor: em busca de parâmetros cognitivos. In: Pagano, A.; Magalhães, C.; Alves, F. (org.). Competência em Tradução: cognição e discurso. Belo Horizonte: Editora da UFMG, 2005, p. 59-90.

GonÇALVES, José Luiz Vila Real. Repensando o desenvolvimento da competência tradutória e suas implicações para a formação do tradutor. Revista Graphos, v. 17, n. 1, 2015, p. 114-130. Disponível em: http://www.periodicos.ufpb.br/index.php/graphos/ article/view/25053 (Acesso: 20/08/2018).

GÖPFERICH, S. Translationsprozessforschung. Stand-Methoden-Perspektiven. Tübingen: Narr, 2008.

GÖPFERICH, S. Towards a model of translation competence and its acquisition: the longitudinal study TransComp. In: GÖPFERICH, S.; JAKOBSEN, A. L.; MEES, I. M. (orgs). Behind the Mind: Methods, models and results in translation process research. Copenhagen: Samfundslitteratur, 2009, p. 11-37.

HuRTADO AlbiR, A. A aquisição da competência tradutória: aspectos teóricos e didáticos. In: Pagano, A.; Magalhães, C.; Alves, F. Competência em Tradução: cognição e discurso. Belo Horizonte: Editora da UFMG, 2005, p. 19-57.

LiPARINI CAMPOS, T. O Efeito da Pressão de Tempo na Realização de Tarefas de Tradução: considerações sobre o produto tradutório. Revista da ABRALIN, v. 7, 20108, p. 223-241.

LIPARINI CAMPOS, T. O efeito do uso de um sistema de memória de tradução e da pressão de tempo no processo cognitivo de tradutores profissionais. Tese de Doutorado em Linguística Aplicada. Faculdade de Letras da Universidade Federal de Minas Gerais/Poslin, Belo Horizonte, 2010.

LIPARINI CAMPOS, T.; BRAGA, C.N.O.; LEIPNITZ, L. Subcompetência sobre Conhecimentos em Tradução: resultados da primeira fase de um estudo longitudinal. Revista Graphos, v. 17, 2015, p. 131-145. Disponível em: http://www.periodicos.ufpb.br/index.php/graphos/article/view/25054/13708 (Acesso: 20/08/2018).

LIPARINI CAMPOS, T.; LEIPNITZ, L. Competência Tradutória: o desenvolvimento da subcompetência sobre conhecimentos em tradução. Domínios de Lingu@gem, v. 11, 2017, p. 1727-1745. Disponível em: http://www.seer.ufu.br/index.php/dominiosdelinguagem/article/view/37449/21497 (Acesso: 20/08/2018).

LiPARINI CAMPOS, T.; LEIPNITZ, L.; BRAgA, C.N.O. Avaliação da Qualidade da Tradução: resultados da primeira fase de um estudo longitudinal sobre a aquisição da competência tradutória. DELTA. Documentação de Estudos em Linguística Teórica e Aplicada, v. 33, 2017, p. 1323-1352. Disponível em: https://revistas.pucsp.br/index.php/delta/article/view/36522 (Acesso: 20/08/2018).

LORENZO, M.P. Es posible La traducción inversa? Resultados de un experimento sobre traducción profesional a una lengua extranjera. In: HANSEN, Gide. (org.). Empirical translation studies: process and product. Copenhagen: Samfundslitteratur, 2002, p. 85124.

PACTE. Acquiring translation competence: hypotheses and methodological problems in a research project. In: BEEBY, A.; ENSINGER, D.; PRESAS, M. Investigating Translation. Amsterdam: John Benjamins, 2000, p. 99-106.

PACTE. Building a translation competence model. In: Alves, F. (org.). Triangulating Translation: Perspectives in process-oriented research. Amsterdam/Philadelphia: John Benjamins, 2003, p. 43-66. 
Leipnitz, L.; Pickbrenner, M.B. - Das Betrachten des Übersetzungsprozesses

PACTE. Primeros resultados de un experimento sobre la Competencia Traductora. In: Actas del II Congresso Internacional de la AIETI (Asociación Ibérica de Estudios de Tradución e Interpretación) Información y documentación, Madrid: Publicaciones de la Universidad Pontificia Comillas, 2005a, p. 573-587 (CD-ROM).

PACTE. Investigating Translation Competence: Conceptual and Methodological Issues. Meta, v. 50, n. 2, 2005b, p. 609-619.

PACTE. First Results of a Translation Competence Experiment: 'Knowledge of Translation' and 'Efficacy of the Translation Process'. In: KEARNS, J. Translator and Interpreter Training: Issues, Methods and Debates. London: Continuum International Publishing Group, 2008, p. 104-126.

PACTE. Results of the Validation of the PACTE Translation Competence Model: Translation Problems and Translation Competence. In: Alvstad, C.; HiLd, A.; Tiselius, E. Methods and Strategies of Process Research: integrative approaches in translation studies. Amsterdam: John Benjamins, 2011b, p. 317-343.

PACTE. First Results of PACTE Group's Experimental Research on Translation Competence Acquisition: The Acquisition of Declarative Knowledge of Translation. MonTI. Monografías de Traducción e Interpretación, Alicanti, 2014, p. 85-115. Disponível em: https://www.redalyc.org/pdf/2651/265134676003.pdf (Acesso: 20/11/2019).

RotHE-NEVES, R. Características cognitivas e desempenho em tradução: investigação em tempo real. Tese de Doutorado em Linguística Aplicada. Faculdade de Letras da Universidade Federal de Minas Gerais/Poslin, Belo Horizonte, 2002.

VASCONCELLOS, M.L. Can the translator play with the system too? A study of thematic structure in some Portuguese translations. Cadernos de Tradução, Florianópolis, v. 2, 1997, p. 137-184.

\section{Quellen der in der Studie in Leipzig benutzten Texte}

Azevedo, A.; Ferreira, M.A.F.; AraúJo, A.A. Consumo de ansiolíticos benzodiazepínicos: Uma correlação entre dados do SNGPC e indicadores sociodemográficos nas capitais brasileiras. Ciência \& Saúde Coletiva, v. 21, n. 1, 2016, p. 149. Disponível em: http://www.cienciaesaudecoletiva.com.br/edicoes (Acesso: 25/04/2018).

OvERBECK, P. Gute Erfahrungen in Sachen Haltbarkeit von Transkatheter-Herzklappen. Quelle: | TAVI| Nachrichten. In: Springer Medizin. Langzeitdaten aus einem deutschen Herzzentrum. Disponível em: https://www.kardiologie.org/tavi/gute-erfahrungen-insachen-haltbarkeit-von-transkatheter-herzkla/15767922 (Acesso: 25/04/2018).

SORKIN, D.; RoOK, K.S.; LU, J.L. Loneliness, lack of emotional support, lack of companionship, and the likelihood of having a heart condition in an elderly sample. Annals of Behavioral Medicine, v. 24, n. 4, 2002, p. 290-298. Disponível em: https://link.springer.com/article/10.1207/S15324796ABM2404_05 (Acesso: 25/04/2018). 